\title{
Hepatic manifestations of drug reaction with eosinophilia and systemic symptoms syndrome
}

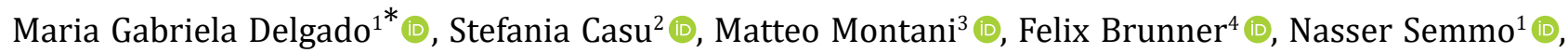 \\ Annalisa Berzigotti ${ }^{1,5}$ (D) Jean François Dufour ${ }^{1,5^{*}}$ (1) \\ ${ }^{1}$ Hepatology, University Clinic of Visceral Surgery and Medicine, Inselspital, University Hospital Bern, 3010 Bern, Switzerland \\ ${ }^{2}$ ASST Santi Paolo e Carlo, Hepatology and Gastroenterology Unit, 20153 Milano, Italy \\ ${ }^{3}$ Institute of Pathology, Inselspital, University Hospital Bern, 3008 Bern, Switzerland \\ ${ }^{4}$ Clinic of Gastroenterolgy and Hepatology, Bürgerspital Solothurn, 4500 Solothurn, Switzerland \\ ${ }^{5}$ Hepatology, Department of Biomedical Research, University of Bern, 3008 Bern, Switzerland
}

*Correspondence: Jean François Dufour, Hepatology, Department of Visceral Surgery and Medicine, Inselspital, Bern University Hospital, Freiburgstrasse 4, 3010 Bern, Switzerland. jean-Francois.Dufour@insel.ch; Maria Gabriela Delgado, Hepatology, Department of Visceral Surgery and Medicine, Murtenstrasse 35, 3010 Bern, Switzerland. mariagabriela.delgado@insel.ch

Academic Editor: Amedeo Lonardo, Azienda Ospedaliero-Universitaria di Modena, Italy

Received: October 28, 2020 Accepted: March 17, 2021 Published: April 30, 2021

Cite this article: Delgado MG, Casu S, Montani M, Brunner F, Semmo N, Berzigotti A, et al. Hepatic manifestations of drug reaction with eosinophilia and systemic symptoms syndrome. Explor Med. 2021;2:122-34. https://doi.org/10.37349/ emed.2021.00037

\begin{abstract}
Drug reaction with eosinophilia and systemic symptoms (DRESS) syndrome is a potentially life-threatening drug reaction, which can affect multiple organs. Patients with DRESS syndrome and hepatic manifestations may present alterations ranging from mild hepatitis to acute liver failure. The diagnosis might be difficult, and the management of these patients is challenging. This report analyzes a series of five cases reporting the clinical presentation, which ranged from acute hepatitis to liver failure, and discussed their treatment.
\end{abstract}

\section{Keywords}

Drug reaction with eosinophilia and systemic symptoms, liver manifestations, diagnosis, treatment

\section{Introduction}

Drug-induced hypersensitivity reaction with systemic symptoms and eosinophilia syndrome is an infrequent but potentially lethal cause of liver injury $[1,2]$. The first description of cases was in the 1950 s related to the use of anticonvulsants [3, 4]. In 1996 Bocquet et al. [1] introduced the term drug rash with eosinophilia and systemic symptoms (DRESS) syndrome. After that, the term "rash" was replaced by "reaction" because skin involvement is not mandatory for the diagnosis [1]. The terminology of this syndrome has changed over time, including other names such as drug-induced hypersensitivity syndrome (DIHS), drug-induced delayed multiorgan hypersensitivity syndrome (DIDMOHS), or anticonvulsant hypersensitivity syndrome (HSS) [5].

The clinical pattern is diverse and includes variable cutaneous and systemic symptoms such as skin rash, fever, enlarged lymph nodes, hypereosinophilia and involvement of at least one organ among liver, lung, kidney, heart, pancreas, and nervous system. Based on these observations, a diagnostic scoring system has (C) The Author(s) 2021. This is an Open Access article licensed under a Creative Commons Attribution 4.0 International License (https://creativecommons.org/licenses/by/4.0/), which permits unrestricted use, sharing, adaptation, distribution and reproduction in any medium or format, for any purpose, even commercially, as long as you give appropriate credit to the original author(s) and the source, provide a link to the Creative Commons license, and indicate if changes were made. 
been developed (RegiSCAR score) [6, 7]. The severity of this syndrome is related to systemic involvement, which can result in multi-organ failure. The liver is the most frequently affected visceral organ in patients with DRESS syndrome [2, 8-10]. Hepatic involvement has been described in up to 75-95\% of patients, which can range from a slight elevation of transaminases to liver failure, being associated with a mortality of around $10 \%[1,8,11-14]$. It has been postulated that the clinical features may be related to the class of drug taken [7].

Severe DRESS syndrome cases are challenging and usually present with significant alteration of liver enzymes or even liver failure. For this reason, this syndrome requires an extensive workup to rule out other possible causes of acute hepatitis and acute liver failure. Most of these patients respond to steroid treatment, but a small percentage may require rescue therapy with liver transplantation [15]. We present a series of five cases of DRESS syndrome and liver involvement.

\section{Case report}

Case 1

A 34-year-old man was referred to the emergency department for cough, fever, and a severely impaired liver function test. The symptoms started three weeks before the admission. After seven days of treatment with amoxicillin/clavulanic acid ( $3 \mathrm{~g} /$ day), paracetamol ( $4 \mathrm{~g} /$ day), and metamizole ( $4 \mathrm{~g} /$ day), he recovered completely. Two weeks later, fever $\left(39-40^{\circ} \mathrm{C}\right)$, and cough recurred, and the patient was admitted to the hospital with the suspicion of sepsis and liver failure. On admission, the patient was febrile $\left(39.3^{\circ} \mathrm{C}\right)$, his pulse regular at 99 beats per minute, his blood pressure was $107 / 68 \mathrm{mmHg}(1 \mathrm{mmHg}=0.133 \mathrm{kPa})$, respiratory rate was 20 per minute, and his oxygen saturation was normal. The examination of the skin, heart, and abdomen was unremarkable. Lung examination displayed basal percussion dullness. There were no skin or mucosal lesions or palpable lymphadenopathy. The neurologic exam was normal. The main laboratory findings are shown in Table 1. The C-reactive protein (CRP) was $337 \mathrm{mg} / \mathrm{L}$, the procalcitonin $5.59 \mu \mathrm{g} / \mathrm{L}(<0.1 \mu \mathrm{g} / \mathrm{L}$ ) and the D-dimer-test was markedly elevated (11,590 $\mu \mathrm{g} / \mathrm{L}$; standard value: $\leq 500 \mu \mathrm{g} / \mathrm{L})$, Fibrinogen and thrombocytes were normal. Toxicological screening, including paracetamol, was negative. Urine and blood cultures were repeatedly negative. Screening tests for autoimmune liver disease and standard virologic and parasitological tests were negative (Table 1).

Empiric antibiotic therapy with piperacillin/tazobactam was initiated. Computed tomography (CT) scan was performed and showed interstitial pneumonia, bilateral pleural effusions, and ascites. The patient further deteriorated, worsening leukocytosis with $25.8 \mathrm{G} / \mathrm{L}$ (eosinophils: $10.6 \%$ ) and CRP of $285 \mathrm{mg} / \mathrm{L}$ at day 5 of the antibiotic therapy. A transjugular liver biopsy was performed. The histology showed an extensive liver cell necrosis with marked eosinophilic infiltrates, indicating drug-induced hepatitis (Figure 1). The liver histology, the eosinophilia, and the persistence of fever despite antibiotic treatment raised the high suspicion of DRESS syndrome. RegiSCAR score was 4 (probable case). Therefore, piperacillin/tazobactam was discontinued after five days, and prednisone $1 \mathrm{mg} / \mathrm{kg}$ was started. The patient had a rapid clinical and laboratory improvement after initiation of the treatment. Interestingly, ten days after the reduction of steroids, the patient developed a non-pruritic, non-painful rash over the patient's chest, abdomen, and back in parallel with fever and rising of the eosinophils ( $18 \mathrm{G} / \mathrm{L})$. These findings were interpreted as a DRESS syndrome flare, so the dose of steroids was increased. Within the first $24 \mathrm{~h}$, he ameliorated significantly. The patient was discharged after two weeks, and prednisone was further slowly tapered (by $5 \mathrm{mg}$ per week). Repeated polymerase chain reactions (PCRs) in the follow-up showed no signs of HHV-6 or HHV-7 reactivation (12 days and 4 weeks). At follow-up, the patient normalized the entire laboratory tests and remained asymptomatic.

\section{Case 2}

A 61-year-old man with a history of polyarthritis under therapy with prednisone $5 \mathrm{mg}$ and sulfasalazine $500 \mathrm{mg}$ Tid was admitted due to persistent fever and rash. The patient reported that he had already suffered from fever and progressive asthenia 16 days before admission. At that time, he had received ambulant treatment with ciprofloxacin for suspected urinary tract infection. After five days of treatment, the fever persisted, and a diffuse rash appeared. The patient was admitted to the hospital, and treatment with amoxicillin/clavulanic and prednisone $25 \mathrm{mg}$ was started. The workup showed leukocytosis, light eosinophilia without atypical lymphocytes. Liver tests were elevated and INR prolonged. Screening for autoimmune liver 
disease and standard virologic and parasitological tests were negative (Table 1). A Staphylococcus aureus grew in the blood culture and switched the antibiotic to vancomycin. The abdominal ultrasound did not show signs of liver or spleen enlargement. RegiSCAR score was 4 (probable case). On day 3 of hospitalization, the patient developed liver failure, and a transjugular liver biopsy was performed. The histology showed panacinar necrosis/apoptosis of the lobular zones 2 and 3, cholestasis, damage to the remaining hepatocyte population, lymphocytic and plasma cellular reactions in the portal fields with portal eosinophilic infiltrated. The findings were concluded as compatible with DRESS syndrome (Figure 2). Following clinical worsening, an urgent LT was performed. The biopsy of the explant confirmed the diagnosis of DRESS syndrome (Figure 3). The patient received treatment with prednisone, mycophenolate mofetil, and cyclosporine. The alteration of the hepatic profile persisted. A percutaneous liver biopsy was performed, which showed signs of rejection (Banff criteria: 6 points). Treatment with a high dose of methylprednisolone was started. Sixteen days after LT, the patient developed unspecific symptoms with neurologic impairment and metabolic acidosis. Within hours, he presented cardiorespiratory arrest that did not respond to cardiovascular resuscitation. The liver histology on the autopsy showed a pattern compatible with DRESS syndrome with moderated eosinophilic infiltrate and diffuse necrosis.

\section{Case 3}

A 60-year-old man was treated for years with phenytoin and levetiracetam for epilepsy. He was admitted to the hospital due to progressive asthenia, fever, cough, dark urine, rash, and severely elevated liver tests with clinical suspicion of DRESS syndrome. On admission, the patient was febrile $\left(39.9^{\circ} \mathrm{C}\right)$, with tachycardia, and normal blood pressure $(115 / 70 \mathrm{mmHg})$. A diffuse erythematous maculopapular rash was present on his face, upper torso, back, and upper and lower extremities without mucosal involvement. Cardiovascular, lungs, abdomen, and neurological examination were normal. No lymphadenopathy was palpable. The laboratory findings showed leukocytosis, eosinophilia, and atypical lymphocytes. Liver tests were elevated (Table 1). INR was prolonged, and factor V was in the normal range. The CRP was $134 \mathrm{mg} / \mathrm{L}$. Toxicological screening, including paracetamol, was negative. Urine and blood cultures were repeatedly negative. Screening for autoimmune liver disorders, virologic and parasitological tests were negative (Table 1). Abdominal ultrasound was normal. A skin biopsy was performed and showed a superficial and deep perivascular dermatitis with interface changes and eosinophilic infiltration. The liver histology showed massive eosinophil infiltrates (Figure 4). RegiSCAR score was 6 (definite case). Levetiracetam was stopped, and the patient started $1 \mathrm{mg} / \mathrm{kg}$ prednisone daily. All the symptoms and laboratory findings rapidly improved. The patient was discharged with tapering prednisone therapy (by $10 \mathrm{mg}$ weekly; at the $20 \mathrm{mg}$ dose: reduction by $5 \mathrm{mg}$ per week). During the follow-up, the patient remained asymptomatic.

\section{Case 4}

A 62-year-old woman developed after 15 days with cefuroxime treatment (7 days) a diffuse maculopapular rash. The patient was admitted by clinical suspicion of DRESS syndrome (rash, elevated eosinophils, and abnormal liver test). At the moment of admission, the vital signs were under the normal range. Diffuse erythematous maculopapular rash was present on her upper torso, back, and upper and lower extremities. No mucosal involvement was described. Cardiovascular, lungs, abdomen, and neurological examination were normal. There was no palpable lymphadenopathy. The laboratory tests showed normal leukocytes, eosinophilia without atypical lymphocytes. Liver tests were elevated, but the liver function was preserved (Table 1). Urine and blood cultures were negative. The toxicological, virologic, and parasitological tests were negative. The autoimmune liver screening did not show abnormalities (Table 1). The sonographic findings of the abdomen were also normal. Liver biopsy demonstrated portal infiltrates with eosinophilic granulocytes, low portal edema, minor reactive changes in the bile duct, and focal accentuated canalicular cholestasis, and low predominantly microvesicular centrally emphasized steatosis (5-10\% of hepatocytes, Figure 5). All these findings were compatible with DRESS syndrome. RegiSCAR score was 4 (probable case). Treatment with $40 \mathrm{mg}$ daily of prednisone was started. All the symptoms and laboratory tests rapidly improved. The patient was discharged with gradual tapering of steroids therapy (by $5 \mathrm{mg}$ per week). During the follow-up, she remained asymptomatic. 


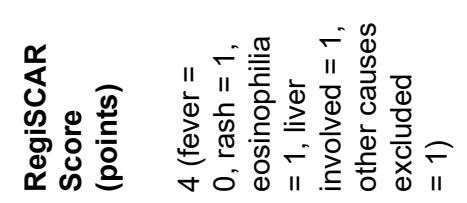

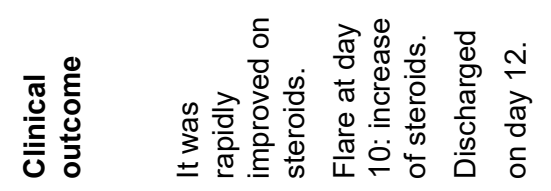

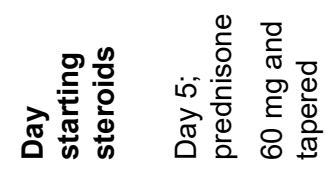

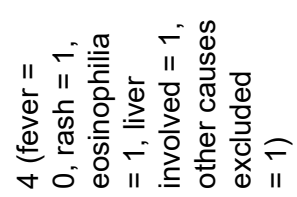

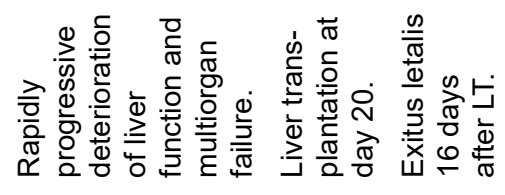

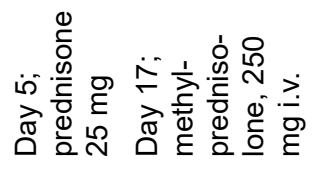

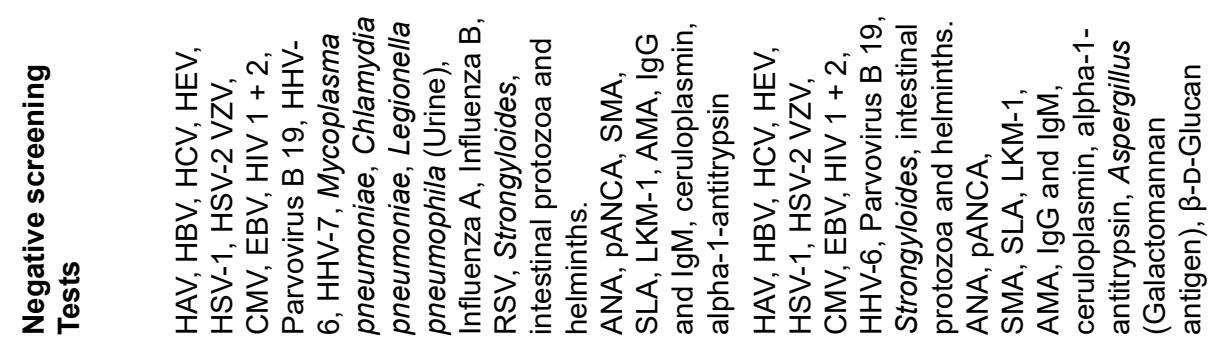

$\stackrel{\alpha}{\stackrel{\alpha}{ }}$

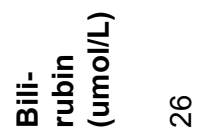

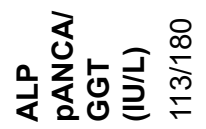

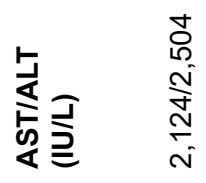

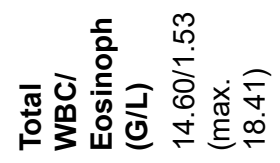

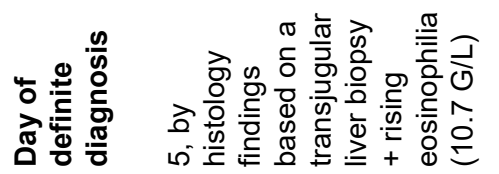

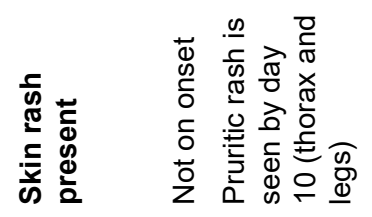

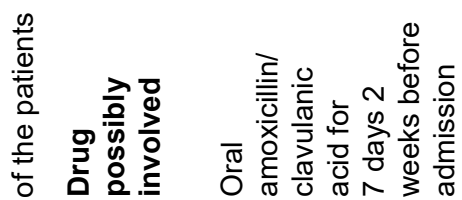

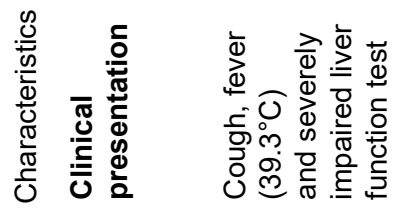

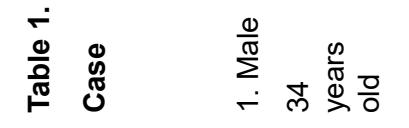

$\stackrel{\infty}{\circ}$

q

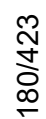

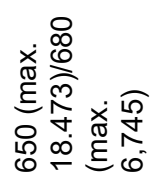

$\infty$
$\stackrel{0}{0}$

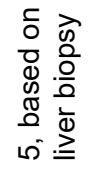

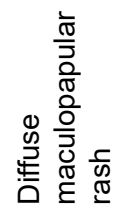

$\frac{1}{\overline{0}}$
$\frac{\pi}{0}$
$\frac{0}{\bar{L}}$
$\frac{0}{0}$
$\frac{0}{0}$

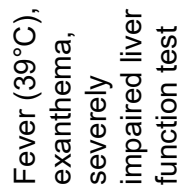

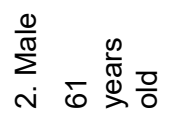




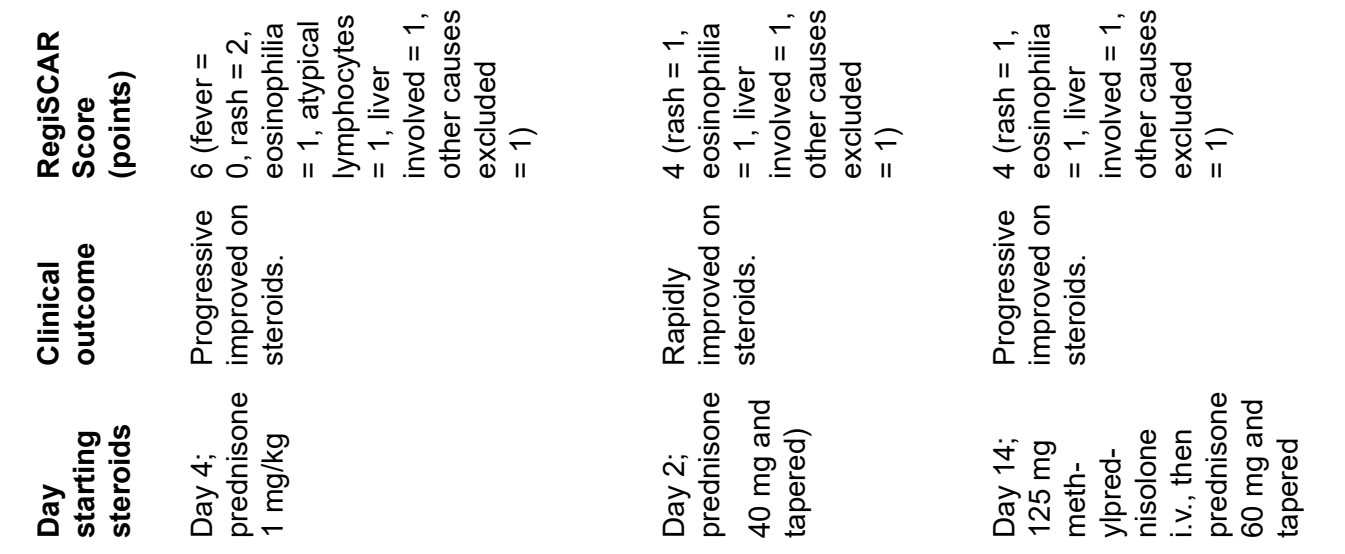

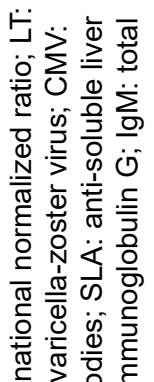

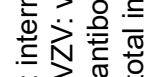

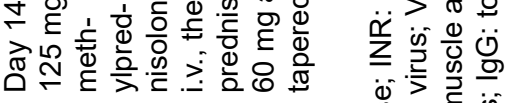

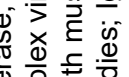

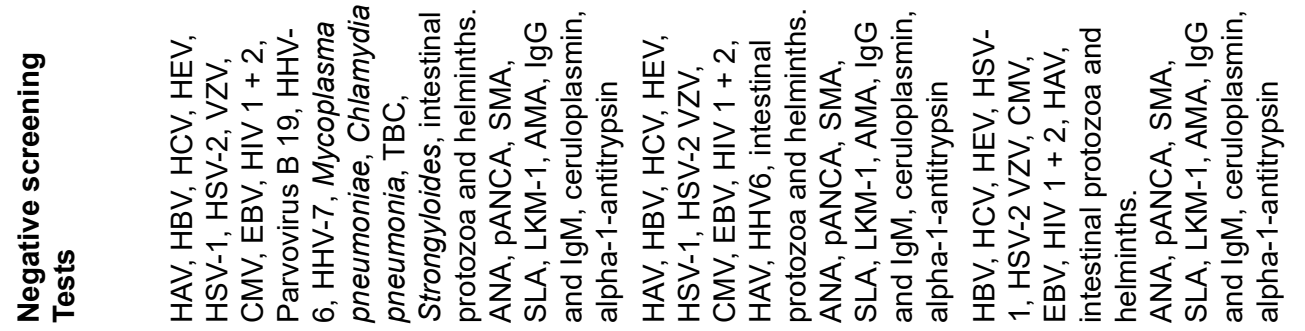

$\underline{\underline{\alpha}} \stackrel{\circ}{\check{r}}$

立 竞高

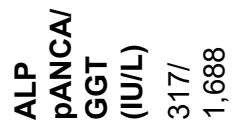

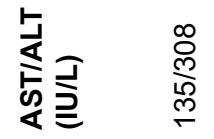

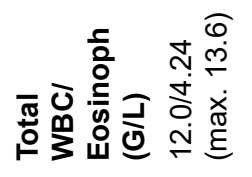

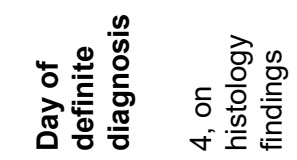

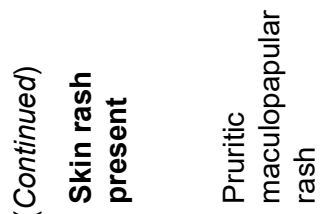

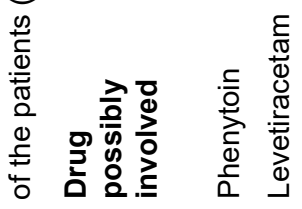

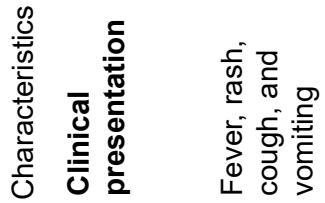

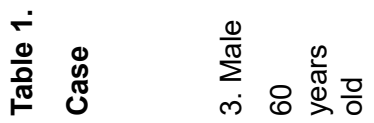

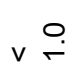

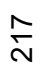

品

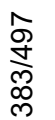

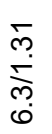

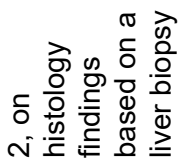

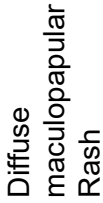

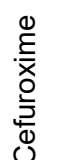

름

离

紊

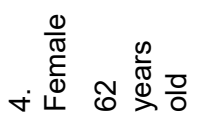

$\stackrel{\Upsilon}{\sim}$

$\frac{\infty}{\frac{9}{2}}$

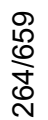

$\infty$

$\underset{\substack{\infty \\ \infty}}{\stackrel{5}{\infty}}$

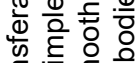

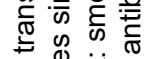

ते

त्र 5 क

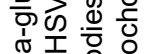

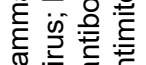

○)

它山岕焉芒

की

तٓ

을 岂芒

동

言了 o

은

잉

这离宁

बं山ें

造的主章

元 $\frac{2}{3}$ 的

일일

素

类

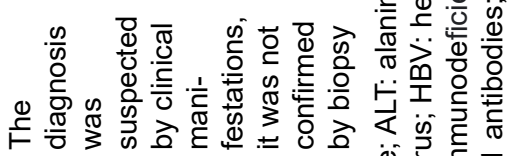

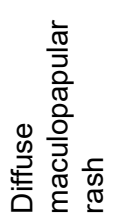

造

吾

흘 옹융

है

정

:

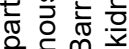

of

的密产要

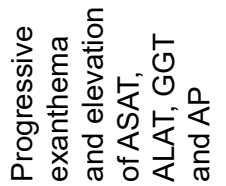

in

这臭

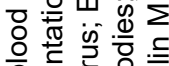

응 흥 홍을 흥

药

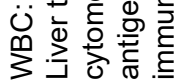




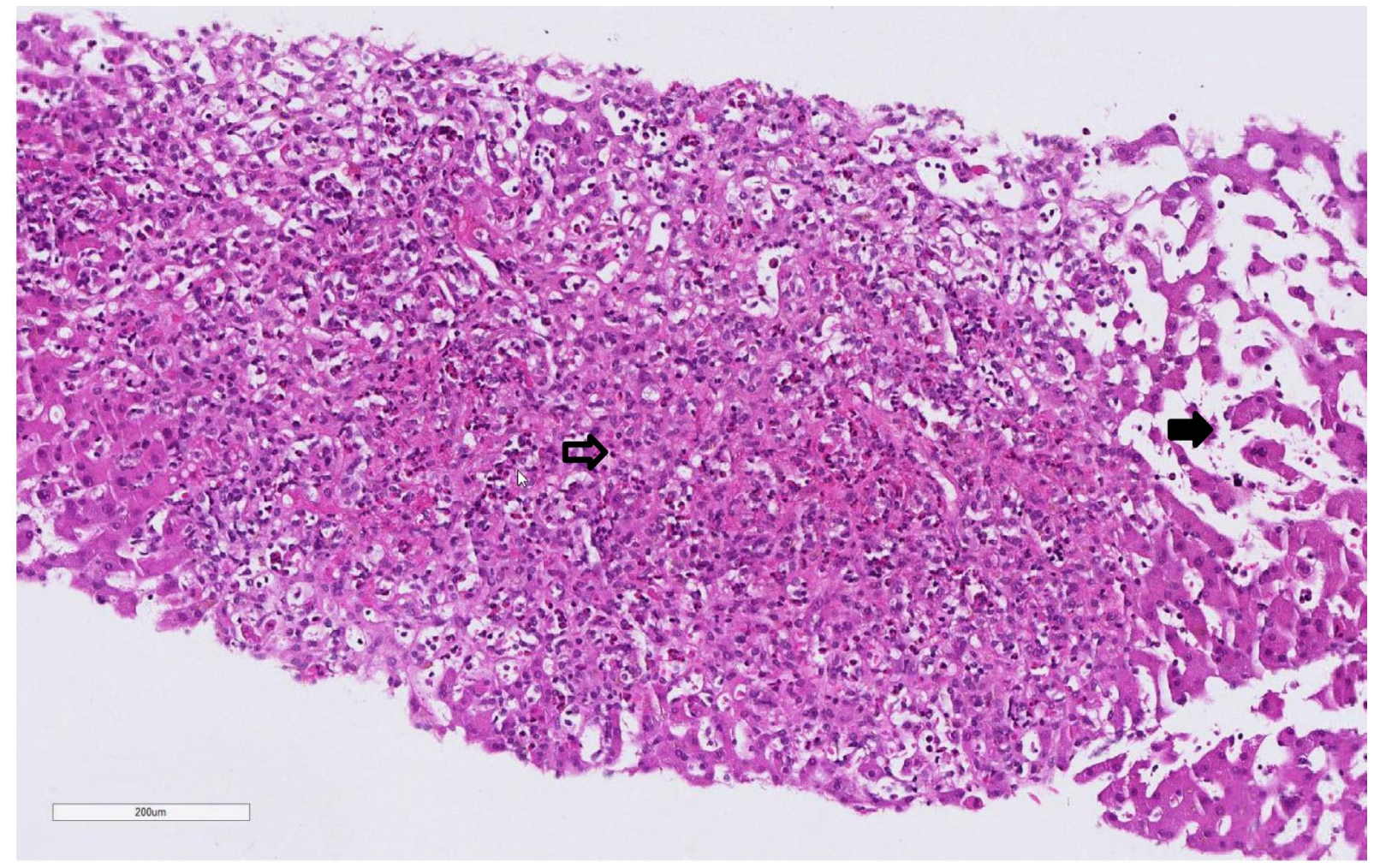

Figure 1. Case 1 liver biopsy: extensive centrilobular (zone 3 to 2 ) necrosis with fiber collapse and prominent eosinophilia (empty arrow). Presence of sinusoid dilatation (full arrow, hematoxylin-eosin, 20x)

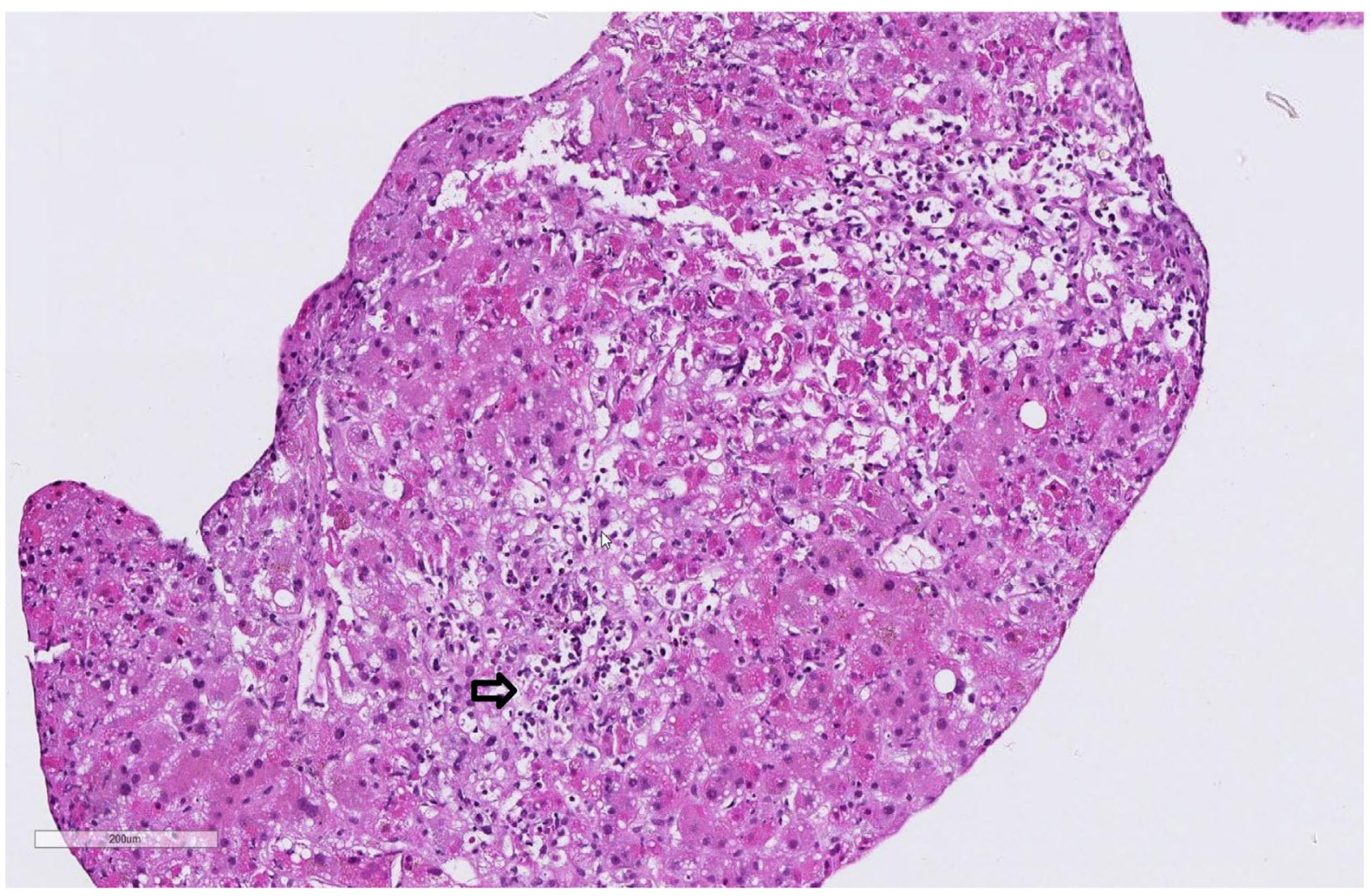

Figure 2. Case 2 liver biopsy: portal fields has expanded, due to a mixed cell infiltrate dominated by lymphocytes and eosinophils. Extensive interface lesions. Bile duct alterations and florid endothelial changes (arrow, hematoxylin-eosin, 20x) 


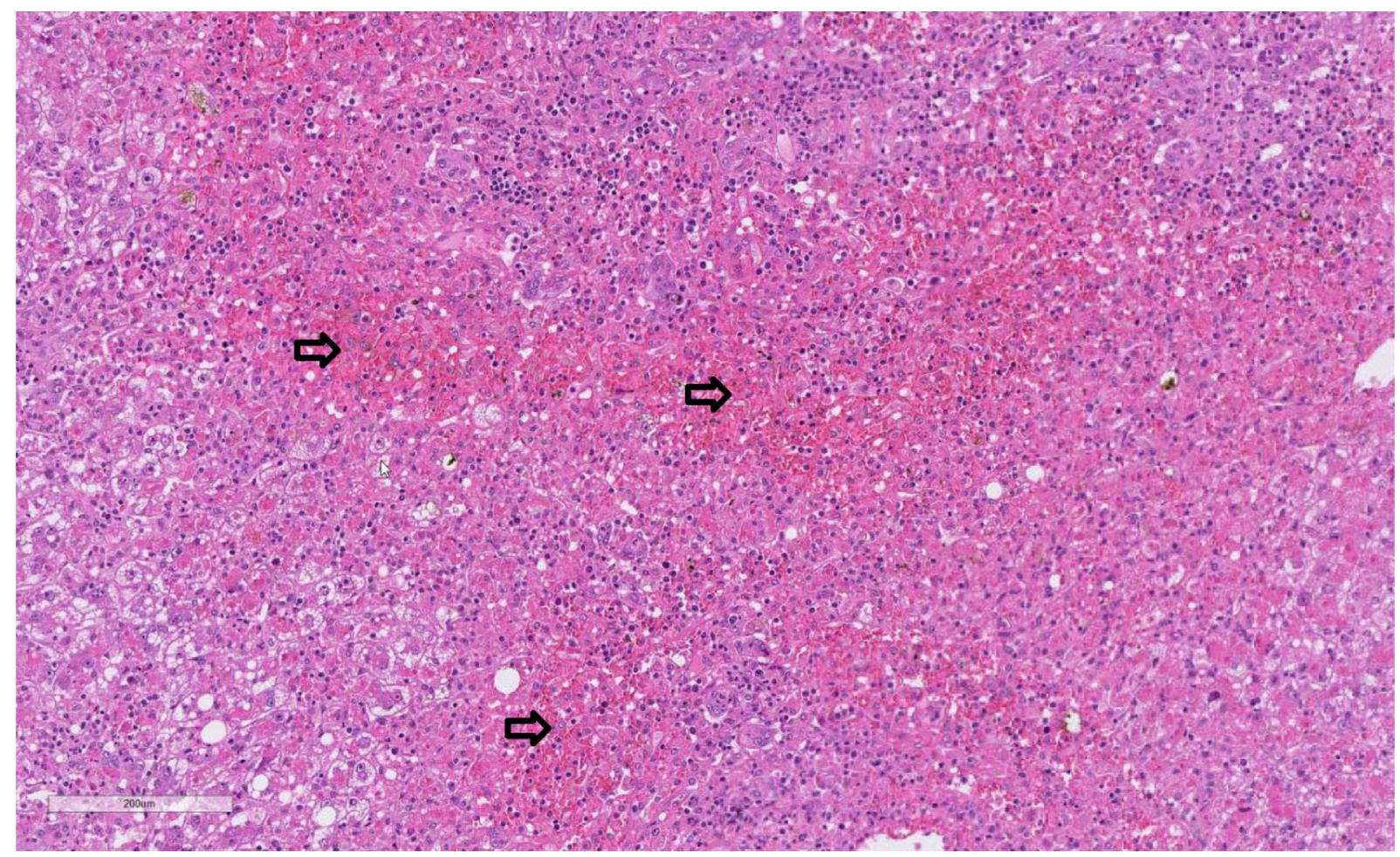

Figure 3. Case 2 biopsy of liver explant: liver explant (I; 1,764 $\mathrm{g}$ heavy) with extensive panacinar necrosis/apoptosis of the lobular zones 2 and 3 (especially pericentral, arrows), cholestasis, damage of the remaining hepatocyte population (with ballooning) as well as lymphocytic and plasmacellular reactions in the portal fields (hematoxylin-eosin, 20x)

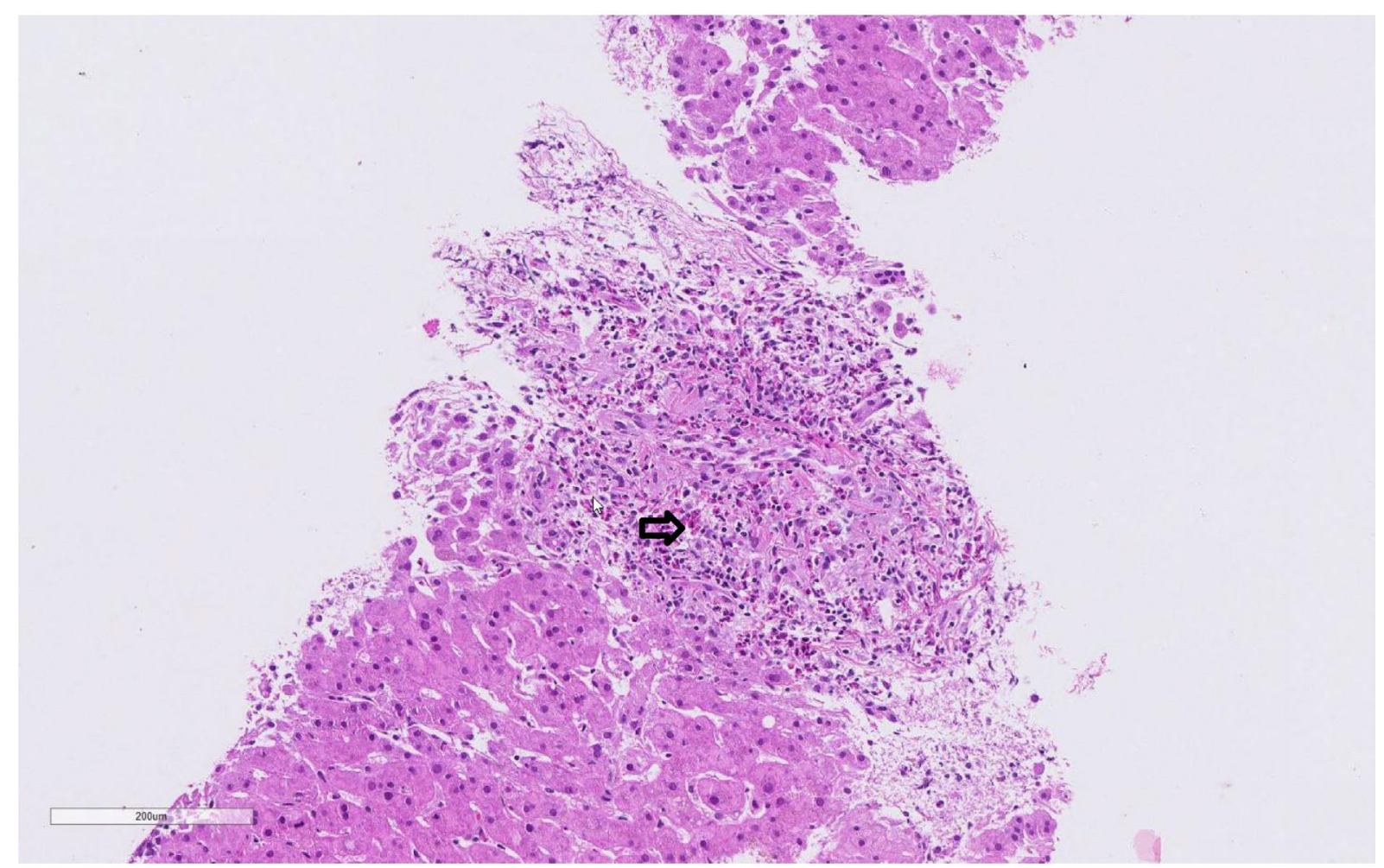

Figure 4. Case 3 liver biopsy: eosinophilic portal field infiltrate (arrow, hematoxylin-eosin, 20x) 


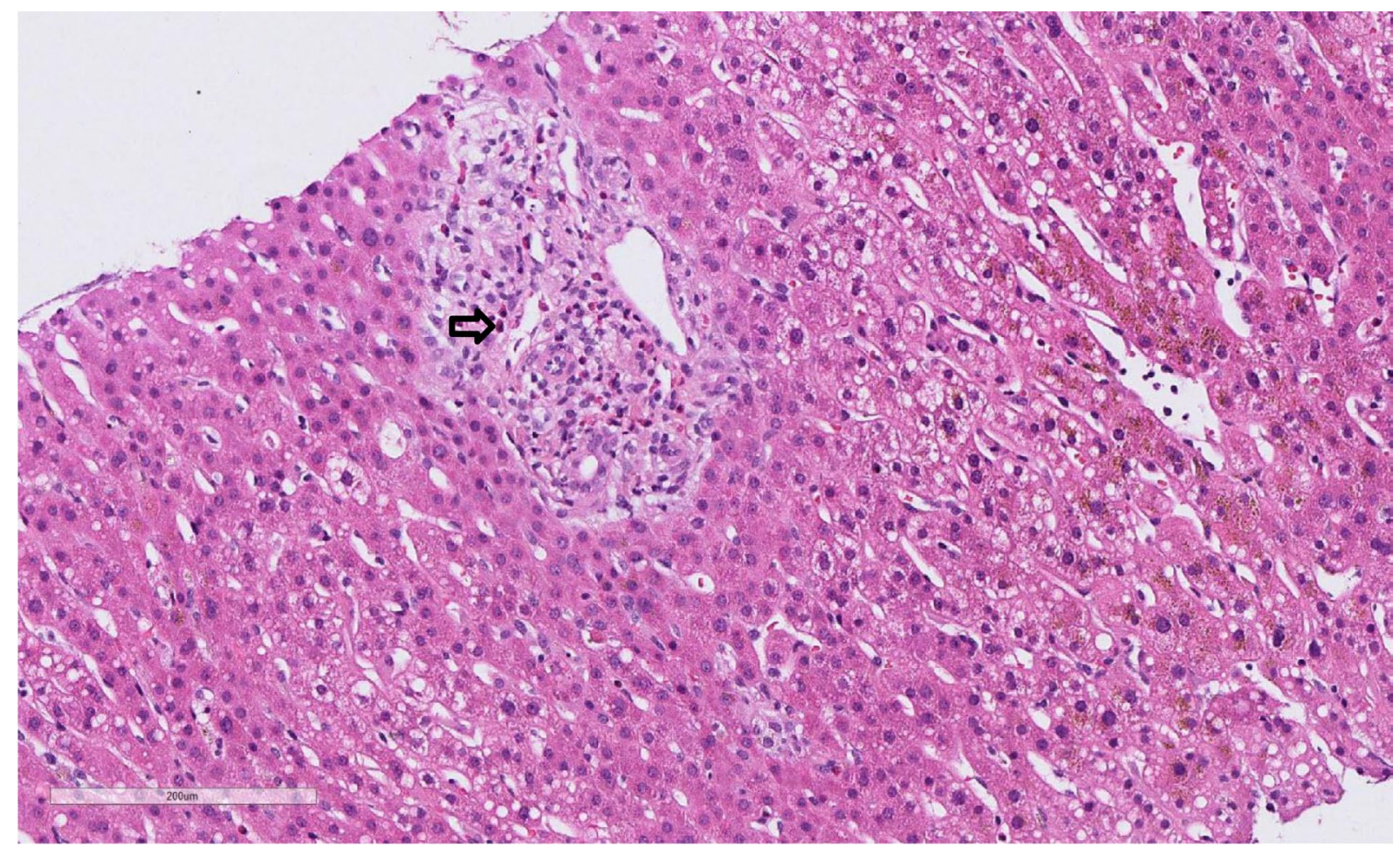

Figure 5. Case 4 liver biopsy: eosinophilic portal field infiltrate (arrow, hematoxylin-eosin, 20x)

\section{Case 5}

A 65-year-old man was treated since February 2018 with lamotrigine and levetiracetam for structural epilepsy without proper compliance. In March 2019, the patient was admitted in the hospital after a car accident. During the hospitalization, the level of lamotrigine in serum was not detectable. Lamotrigine was reinitiated. After four weeks from discharge, the patient was readmitted, presenting a non-itching progressive exanthema for two weeks. At the moment of admission, the vital signs were under normal range. Diffuse erythematous maculopapular rash was present with swelling of lips, hands, and feet. Cardiovascular, lungs, abdomen, and neurological examination were normal. There was no palpable lymphadenopathy. The laboratory tests showed normal leukocytes, eosinophilia, and lymphocytosis. Liver tests were elevated, but the liver function was preserved. The toxicological, virologic, and parasitological tests were negative (Table 1). The abdominal ultrasound was normal. The patient was admitted by clinical suspicion of DRESS syndrome (rash, elevated eosinophils, and abnormal liver test). The RegiSCAR score was 4 (probable case). He received a single dose of prednisolone i.v. $(125 \mathrm{mg})$ in the emergency room, then started treatment with $60 \mathrm{mg}$ of prednisone daily. The patient was discharged with gradual tapering of steroids therapy (by $10 \mathrm{mg}$ per week; at the $20 \mathrm{mg}$ dose: reduction by $5 \mathrm{mg}$ per week). The symptoms and laboratory tests progressively improved.

\section{Discussion}

DRESS syndrome is an idiosyncratic adverse drug reaction characterized by a maculopapular rash, high fever, lymphadenopathy, visceral involvement, eosinophilia, and presence of atypical lymphocytes. An incidence ranging from $1 / 1,000$ to $1 / 10,000$ patients/year per drug exposures has been reported $[16,17]$. The annual prevalence ranges from 2.8 [18] to 9.6 cases per 100,000 inpatients [13]. DRESS syndrome is a potentially fatal condition with mortality approximately of $10 \%[2,12]$. This syndrome is usually characterized by lateonset (up to 10 weeks after the first drug intake), but there have been described cases developing symptoms within first days after the drug exposure [14]. Flares after drug discontinuation have also been described [10]. Many drugs have been related to this syndrome and the most frequent associated are anticonvulsants, allopurinol, anti-tuberculosis drugs, antibiotics, sulfonamides, non-steroidal anti-inflammatory drugs (NSAIDs), and diuretics [2, 12, 14, 19-21]. The drugs involved in our cases were amoxicillin/clavulanic, phenytoin, levetiracetam, lamotrigine, ciprofloxacin, and cefuroxime. Multiple hypotheses have been proposed to explain the pathogenesis of DRESS syndrome, such as genetic predisposition in important 
genes for drug metabolism and reactivation of viral infections such as HHV-6 and 7, cytomegalovirus (CMV), Epstein-Barr virus (EBV) [2,14, 22]. A study involving 100 patients demonstrated that HHV-6 viremia usually appears between day 10 to day 28, and detection of anti-HHV-6 in serum occurs simultaneously or later. HHV-6 reactivation was associated with poor prognosis [22]. Reactivation of CMV, EBV, and HHV-6 was ruled out by serological antibodies assay and quantitative real-time PCR within two weeks of onset symptoms in four patients in our series. HHV-7 was tested in two patients. Antibodies against HHV-6 and HHV-7 were detected by Immunofluorescence Assay.

One of the most frequent signs described in patients with DRESS syndrome is fever, up to $90 \%$ of recorded patients $[2,6,14,19]$, and usually precedes the skin rash. Pruritus could appear before the skin lesions $[2,6$, $14,19]$. The most common cutaneous manifestation is the diffuse erythematous maculopapular rash $85 \%$ of cases) [19] and usually affects the face, trunk, and upper limbs, extending later to the rest of the body. Facial edema appears in $76 \%$ of cases [19]. Mucosal involvement is reported up to $50 \%$ of cases $[6,10,19]$. Four of our patients presented fever at the moment of diagnosis and maculopapular rash. One of the patients developed the cutaneous lesions after the diagnosis, during the tapering of the steroids, with recurrence of fever, leukocytosis, and eosinophilia, which was interpreted as DRESS syndrome flare. This episode was controlled with an increase in the dose of steroids. The detection of HHV-6 was performed by PCR at day 12 and 4 weeks after the onset of symptoms in our patient. The symptomatology during the flares is usually more severe and improves after the increase of the steroid dose [1, 10].

The most frequent hematological findings described in DRESS syndrome are leukocytosis (95\%), eosinophilia (52-96\%), lymphocytosis (25-52\%), thrombocytosis (19\%), thrombocytopenia (7-25\%), and the presence of atypical lymphocytes (57-63\%) $[6,12,19]$. Patients in our series only presented leukocytes and eosinophilia, and only one atypical lymphocytes without further hematological alteration. One of the patients had elevated levels of D-dimer, and thrombosis was ruled out. It has been reported that patients with DRESS syndrome may have elevated levels of plasma prothrombin fragments, but not D-dimer [23]. Several researchers have suggested a relationship between eosinophil activation, increased vascular permeability, and thrombin generation in these patients [23-25]. Another hematologic alteration described in these patients is hemophagocytic syndrome [9].

Different organs and systems could be affected by DRESS syndrome. Bocquet [1] described that visceral damage could be related to eosinophilia. Studies have described the involvement of a single organ in 35$75 \%[6,13]$ of cases and two or more organs in 56\% [6]. An association between the clinical pattern and the type of drug has been described $[2,6,9,19]$. The liver is the most common organ involved (75-95\%) $[6$, $12,13,20]$. The spectrum of liver damage ranges from mild hepatitis to fulminant liver failure. Elevation of transaminases has been described in up to $90 \%$ of cases [10].

DRESS syndrome could be included in one of the phenotypes of drug-induced liver injury (DILI) [26]. Some authors recommend the DILI severity index to evaluate the degree of liver injury [14, 27]. In our series, two cases were moderate, one was severe, one was mild, and one was fatal, according to this index. The liver biopsy is not mandatory but may provide diagnostic information by matching known patterns of DILI $[28,29]$.

A retrospective multicenter study including 16 patients with severe acute liver damage described an overall survival of 75\% and after liver transplantation of 60\% [15]. Hepatic encephalopathy, Factor V level ( $\leq$ $40 \%$ or higher with a reduction at day 2), and prothrombin time (PT) were the predictors of poor prognosis. Five patients were transplanted, and $60 \%$ presented a recurrence of DRESS syndrome. Mean delay between LT and recurrence was $75 \pm 91$ days [15]. In our series, only two patients had prolongation of the INR and none Factor $\mathrm{V}$ below $40 \%$ or hepatic encephalopathy. Four patients evolved satisfactorily with medical treatment, and one patient received a liver transplant as rescue therapy and died 16 days after. The liver biopsy showed acute rejection (Banff 6) and no DRESS-compatible findings, although signs of recurrent DRESS syndrome were subsequently described in the autopsy. Despite liver transplantation represents the rescue therapy option in some severe cases, the recurrence of the disease is possible regardless of a high dose of immunosuppressants and may lead to graft loss or death. 
Due to the diversity of clinical and laboratory features that DRESS syndrome patients may present, a scoring system denominated RegiSCAR score was created to facilitate the diagnosis. This scoring system classifies the cases as indefinite, probable, possible, or not based on the history of drug exposure and presence of rash, fever, organ dysfunction, enlarged adenopathy, and hematologic abnormalities [6]. The leucocyte transformation/activation test (LTT) could be used to confirm the causative drug of DRESS syndrome. Sensibility of $50 \%$ and specificity of $92 \%$ have been reported in the acute phase of DRESS syndrome [14]. However, both parameters improve dramatically when the test is performed in the recovery phase (4-8 weeks after the reaction). A positive LTT is useful to confirm the diagnosis due to the low rate of false-positive results (only 2\%). However, a negative test cannot exclude the diagnosis [30].

The initial management of DRESS syndrome consists of the withdrawal of the causative drug. Steroids constitute the first therapy line in patients with hepatic manifestation $(0.5-1 \mathrm{mg} / \mathrm{kg}$ daily tapered in $8-12$ weeks) $[14,31]$. The duration of the therapy depends on the severity of the symptoms and the organ involved but should be at least 2 to 3 months [19]. The recently Spanish DRESS syndrome Guidelines recommend in patients with a liver injury, start with oral methylprednisolone $60-120 \mathrm{mg} /$ day or prednisone $40-60 \mathrm{mg} / \mathrm{day}$ and taper by 5-10 mg weekly [14]. In severe cases of hepatitis, human immunoglobulin could be added to the treatment [32]. Other immunosuppressants such as cyclosporine, mycophenolate mofetil, and rituximab have been used in patients who do not have an adequate response to steroids [14, 31]. All our patients received steroids treatment, four improved progressively, and one of them required urgent LT. Patients with DRESS syndrome and severe liver injury without liver transplantation have estimated mortality of 75\% [15].

DRESS syndrome can present with heterogeneous manifestations and can lead to fulminant liver failure. Early recognition, withdrawal of the causative drug and steroids therapy could avoid irreversible damage of the liver. The response to treatment should be evaluated very strictly. The patients who do not show improvement of hepatic function should be referred to a tertiary center for eventual urgent liver transplantation.

\section{Abbreviations}

AMA: antimitochondrial antibodies

ANA: antinuclear antibodies

CRP: C-reactive protein

CT: computerized tomography

CMV: cytomegalovirus

DILI: drug-induced liver injury

DRESS: drug rash with eosinophilia and systemic symptoms

EBV: Epstein-Barr virus

GGT: gamma-glutamyl transferase

HAV: hepatitis A virus

HBV: hepatitis B virus

HCV: hepatitis $C$ virus

HEV: hepatitis E virus

HHV-6: human herpesvirus virus-6

HHV-7: human herpesvirus virus-7

HIV: human immunodeficiency virus

HSV: herpes simplex virus

IgG: total immunoglobulin G

IgM: total immunoglobulin M

INR: international normalized ratio

i.v.: intravenous 
LKM-1: liver kidney microsome type 1 antibodies

LT: liver transplantation

NSAID: non-steroidal anti-inflammatory drugs

pANCA: anti-neutrophil cytoplasmic antibodies

PCRs: polymerase chain reactions

SLA: anti-soluble liver antigen antibodies

SMA: smooth muscle antibodies

VZV: varicella-zoster virus

\section{Declarations}

\section{Author contributions}

The authors confirm contribution to the paper as follows: study conception and design: MGD, FB, AB, JFD. Data collection: MGD, FB, SC, MM. Analysis and interpretation of results: MGD, JFD, AB. Draft manuscript preparation: MGD, JFD, AB, SC, FB. All authors reviewed the results and approved the final version of the manuscript.

\section{Conflicts of interest}

No author has any conflict of interest.

\section{Ethical approval}

All patients provided written consent to the use of their health-related data for research. The hospital ethics committee approved collection of patient information and the study protocol, which was consistent with the principles of the current version of the Declaration of Helsinki, Good Clinical Practice (GCP) guidelines, and local regulatory requirements.

\section{Consent to participate}

All patients provided written consent to the use of their health-related data for research.

\section{Consent to publication}

Not applicable.

\section{Availability of data and materials}

The raw data supporting the conclusions of this manuscript will be made available by the authors, without undue reservation, to any qualified researcher.

\section{Funding}

Not applicable.

\section{Copyright}

(c) The Author(s) 2021.

\section{References}

1. Bocquet H, Bagot M, Roujeau JC. Drug-induced pseudolymphoma and drug hypersensitivity syndrome (Drug Rash with Eosinophilia and Systemic Symptoms: DRESS). Semin Cutan Med Surg. 1996;15:250-7.

2. Husain Z, Reddy BY, Schwartz RA. DRESS syndrome: part I. Clinical perspectives. J Am Acad Dermatol. 2013;68:693.e1-14; quiz 706-8.

3. Chaiken BH, Goldberg BI, Segal JP. Dilantin sensitivity; report of a case of hepatitis with jaundice, pyrexia and exfoliative dermatitis. N Engl J Med. 1950;242:897-8. 
4. Saltzstein SL, Ackerman LV. Lymphadenopathy induced by anticonvulsant drugs and mimicking clinically pathologically malignant lymphomas. Cancer. 1959;12:164-82.

5. Kinoshita Y, Saeki H, Asahina A, Ochiai T, Ijjima M. Drug-induced hypersensitivity syndrome in Japan in the past 10 years based on data from the relief system of the Pharmaceuticals and Medical Devices Agency. Allergol Int. 2017;66:363-5.

6. Kardaun SH, Sidoroff A, Valeyrie-Allanore L, Halevy S, Davidovici BB, Mockenhaupt M, et al. Variability in the clinical pattern of cutaneous side-effects of drugs with systemic symptoms: does a DRESS syndrome really exist? Br J Dermatol. 2007;156:609-11.

7. Peyriere H, Dereure O, Breton H, Demoly P, Cociglio M, Blayac JP, et al. Variability in the clinical pattern of cutaneous side-effects of drugs with systemic symptoms: does a DRESS syndrome really exist? $\mathrm{Br} \mathrm{J}$ Dermatol. 2006;155:422-8.

8. Martinez-Cabriales SA, Shear NH, Gonzalez-Moreno EI. Liver involvement in the drug reaction, eosinophilia, and systemic symptoms syndrome. World J Clin Cases. 2019;7:705-16.

9. Kano Y, Ishida T, Hirahara K, Shiohara T. Visceral involvements and long-term sequelae in drug-induced hypersensitivity syndrome. Med Clin North Am. 2010;94:743-59, xi.

10. Ang CC, Wang YS, Yoosuff EL, Tay YK. Retrospective analysis of drug-induced hypersensitivity syndrome: a study of 27 patients. J Am Acad Dermatol. 2010;63:219-27.

11. Lee T, Lee YS, Yoon SY, Kim S, Bae YJ, Kwon HS, et al. Characteristics of liver injury in drug-induced systemic hypersensitivity reactions. J Am Acad Dermatol. 2013;69:407-15.

12. Chen YC, Chiu HC, Chu CY. Drug reaction with eosinophilia and systemic symptoms: a retrospective study of 60 cases. Arch dermatol. 2010;146:1373-9.

13. Hiransuthikul A, Rattananupong T, Klaewsongkram J, Rerknimitr P, Pongprutthipan M, Ruxrungtham K. Drug-induced hypersensitivity syndrome/drug reaction with eosinophilia and systemic symptoms (DIHS/DRESS): 11 years retrospective study in Thailand. Allergol Int. 2016;65:432-8.

14. Cabanas R, Ramirez E, Sendagorta E, Alamar R, Barranco R, Blanca-Lopez N, et al. Spanish guidelines for diagnosis, management, treatment, and prevention of DRESS syndrome. J Investig Allergol Clin Immunol. 2020;30:229-53.

15. Ichai P, Laurent-Bellue A, Saliba F, Moreau D, Besch C, Francoz C, et al. Acute liver failure/injury related to drug reaction with eosinophilia and systemic symptoms: outcomes and prognostic factors. Transplantation. 2017;101:1830-7.

16. Shiohara T, Kano Y, Takahashi R, Ishida T, Mizukawa Y. Drug-induced hypersensitivity syndrome: recent advances in the diagnosis, pathogenesis and management. Chem Immunol Allergy. 2012;97:122-38.

17. Chiou CC, Yang LC, Hung SI, Chang YC, Kuo TT, Ho HC, et al. Clinicopathological features and prognosis of drug rash with eosinophilia and systemic symptoms: a study of 30 cases in Taiwan. J Eur Acade Dermatol Venereol. 2008;22:1044-9.

18. Wolfson AR, Zhou L, Li Y, Phadke NA, Chow OA, Blumenthal KG. Drug reaction with eosinophilia and systemic symptoms (DRESS) syndrome identified in the electronic health record allergy module. J Allergy Clin Immunol Pract. 2019;7:633-40.

19. Martinez-Cabriales SA, Rodriguez-Bolanos F, Shear NH. Drug reaction with eosinophilia and systemic symptoms (DReSS): how far have we come? Am J Clin Dermatol. 2019;20:217-36.

20. Cacoub P, Musette P, Descamps V, Meyer O, Speirs C, Finzi L, et al. The DRESS syndrome: a literature review. Am J Med. 2011;124:588-97.

21. James J, Sammour YM, Virata AR, Nordin TA, Dumic I. Drug reaction with eosinophilia and systemic symptoms (DRESS) syndrome secondary to furosemide: case report and review of literature. Am J Case Rep. 2018;19:163-70. 
22. Tohyama M, Hashimoto K, Yasukawa M, Kimura H, Horikawa T, Nakajima K, et al. Association of human herpesvirus 6 reactivation with the flaring and severity of drug-induced hypersensitivity syndrome. $\mathrm{Br}$ J Dermatol. 2007;157:934-40.

23. Asero R, Tedeschi A, Riboldi P, Griffini S, Bonanni E, Cugno M. Severe chronic urticaria is associated with elevated plasma levels of D-dimer. Allergy. 2008;63:176-80.

24. Cugno M, Marzano AV, Asero R, Tedeschi A. Activation of blood coagulation in chronic urticaria: pathophysiological and clinical implications. Intern Emerg Med. 2010;5:97-101.

25. Tedeschi A, Kolkhir P, Asero R, Pogorelov D, Olisova O, Kochergin N, et al. Chronic urticaria and coagulation: pathophysiological and clinical aspects. Allergy. 2014;69:683-91.

26. European Association for the Study of the Liver, Clinical Practice Guideline, Panel Members, EASL Governing Board representative. EASL Clinical Practice Guidelines: drug-induced liver injury. J Hepatol. 2019;70:1222-61.

27. Aithal GP, Watkins PB, Andrade RJ, Larrey D, Molokhia M, Takikawa $\mathrm{H}$, et al. Case definition and phenotype standardization in drug-induced liver injury. Clin Pharmacol Ther. 2011;89:806-15.

28. Gasmi B, Kleiner DE. Liver histology: diagnostic and prognostic features. Clin Liver Dis. 2020;24:61-74.

29. Kleiner DE. Histopathological challenges in suspected drug-induced liver injury. Liver Int. 2018;38: 198-209.

30. Cabanas R, Calderon O, Ramirez E, Fiandor A, Caballero T, Heredia R, et al. Sensitivity and specificity of the lymphocyte transformation test in drug reaction with eosinophilia and systemic symptoms causality assessment. Clin Exp Allergy. 2018;48:325-33.

31. Husain Z, Reddy BY, Schwartz RA. DRESS syndrome: part II. Management and therapeutics. J Am Acad Dermatol. 2013;68:709.e1-9; quiz 718-20.

32. Descamps V, Ben Said B, Sassolas B, Truchetet F, Avenel-Audran M, Girardin P, et al. Management of drug reaction with eosinophilia and systemic symptoms (DRESS). Ann Dermatol Venereol. 2010;137:703-8. 